\title{
Si Galileo Galilei hubiera tenido una cámara digital: enseñando ciencias a una generación digital
}

\section{If Galileo Galilei had had a digital camera: teaching science to a digital generation}

Luis Alejandro Andrade Lotero

Indiana University, USA

laandrad@indiana.edu

RESUMEN • El objetivo del presente artículo es mostrar como la cámara digital puede ser una herramienta didáctica para transformar la práctica de educación en ciencias en nuestros salones de clase en el nivel de educación básica. Para ello, se presentan tres casos de proyectos de clase que tienen en común el uso de la cámara digital. Estos casos responden a una teoría de la enseñanza de las ciencias basada en la fundamentación de las prácticas de investigación, cuya herramienta principal es la experimentación. Sin embargo, es indispensable acompañar esta herramienta con nuevas prácticas educativas y nuevas actividades de aprendizaje. De esta manera será posible diseñar nuevas formas para educar a las generaciones que crecen en una sociedad digital y que demandan una nueva cultura de aprendizaje.

PALABRAS CLAVE: constructivismo; educación en ciencias; investigación; nuevas tecnologías de información; prácticas epistémicas.

ABSTRACT $\bullet$ The aim of this paper is to show how the digital camera can serve as a practice-changing educational tool to transform science education in elementary and middle school. We present three class projects which have the use of digital cameras as common denominator. These cases reflect a theory of research-practice-based science education, in which experimentation is the main tool. However, new educational practices and learning activities should accompany this tool. Only by changing the educational practices would we be able to provide new ways to educating our digital-generationkids, which are now demanding a new culture of learning.

KEYWORDS: constructivism; epistemic practices; information technologies; science education. 
Mucho se ha hablado de la necesidad de proponer un papel más activo a los estudiantes en la educación escolar de las ciencias. El objetivo es trascender la enseńanza tradicional y fomentar una perspectiva más constructivista y centrada en el aprendizaje (por ejemplo, Gil Pérez, 1994; Mellado Jiménez, 2003; Moya y Campanario, 1999). Entre otros modelos didácticos, el modelo por investigación ha ganado importancia desde hace varias décadas en el contexto iberoamericano (por ejemplo, Gil Pérez y Valdés Castro, 1996; Moya y Campanario, 1999; Ortega, 2007) y en el anglosajón (por ejemplo, Abd-El-Khalick et al., 2004; Edelson, Gordin y Pea, 1999; National Research Council. Center for Science, 2000; Spronken-Smith et al., 2011). En esta reorientación didáctica son puestas de presente las prácticas de laboratorio y una metodología de solución de problemas por medio de la experimentación (Carrascosa, Pérez, Vilches, yValdez, 2008; Perales Palacios, 2010).

En efecto, la práctica científica lleva asociada la utilización de instrumentos de medición y representación de los fenómenos del mundo (Kozma, 2000), instrumentos que cada vez más dependen de nuevas tecnologías de información (Kozma, 2003). Por esto, esta tendencia ha influenciado la práctica educativa, invitándola a actualizar sus métodos y objetivos en la era digital (Battro y Denham, 1997; Thomas y Brown, 2011). De esta manera, la cámara digital, por ejemplo, la cual captura fotos y video, se muestra como una herramienta didáctica que puede apoyar la transformación de las prácticas educativas en la educación científica de nuestros niños y jóvenes (Carrascosa et al., 2008).

El objetivo del presente artículo es mostrar cómo la cámara digital, de fácil adquisición hoy en día, puede ser una herramienta didáctica para transformar la práctica de educación en ciencias en los salones de clase en el nivel de educación básica. Nuestra intención aquí es mostrar tres casos de proyectos de clase que tienen en común el uso de la cámara digital como herramienta pedagógica en la enseñanza de las ciencias. Se intentarán ilustrar diferentes usos de esta en el trabajo por proyectos con estudiantes, pues los tres ejemplos comparten la misma mirada constructivista de la educación basada en el modelo por investigación. Los tres casos provienen de nuestra experiencia en la enseñanza de las ciencias con profesoras en formación de una licenciatura en educación para la primera infancia en una universidad colombiana.

No obstante, el alcance de esta ilustración no pretende ser exhaustivo, ni tampoco presentar un planteamiento de secuencias didácticas detalladas con este instrumento. Este es, en cambio, un artículo que sirve para ilustrar que sí es posible una educación orientada hacia la experimentación usando herramientas informáticas de bajo costo. Las posibles consecuencias de este uso apuntan indudablemente al aumento de motivación de los estudiantes y a la transformación de las prácticas escolares. Este trabajo, entonces, esperamos sea de interés para docentes en educación primaria y secundaria, así como para investigadores y estudiosos de la didáctica específica de las ciencias en el contexto de las nuevas tecnologías de la información.

El presente artículo está estructurado en tres partes. La primera parte realiza una breve mirada a la teoría de la enseñanza de las ciencias y su fundamentación en una práctica investigativa basada en experimentación, la cual demanda una nueva cultura de aprendizaje. La segunda parte incluye la presentación del estudio de tres casos del uso de la cámara digital en la práctica de educación en ciencias en la escuela. Un primer caso se refiere al estudio de la caída de los cuerpos, es decir, una replicación del experimento clásico de Galileo Galilei, pero sin el plano inclinado, usando en cambio un registro en video y un software de edición. El segundo caso se refiere a la observación del Sol como un cuerpo celeste, replicando las observaciones del origen de la astronomía que se remonta hasta antes de la época de los babilonios. El tercer caso se refiere al uso de la cámara digital por estudiantes de primera infancia, en donde se parte del propio interés del estudiante para capturar el mundo exterior, y así discutir en clase acerca de diversas cuestiones de conocimiento general. En la tercera y última parte se presentan las conclusiones del artículo y se discuten posibles maneras de expandir la investigación en este sentido. 


\section{ILUSTRACIÓN DEL PROBLEMA}

\section{Enseñanza de las ciencias basada en la experimentación y el registro}

Varios modelos didácticos de enseñanza de las ciencias existen a nivel de Primaria y Secundaria (por ejemplo, Mellado Jiménez, 2003; Ortega, 2007). Recientes tendencias no solo resaltan el papel de la experimentación en la comprensión científica, sino que también reconocen que ésta debe estar orientada a la solución de un problema con propósito y finalidad específica en el contexto de los estudiantes (Gil Pérez \&y Valdés Castro, 1996; Jaramillo y García, 2004; Moya y Campanario, 1999).

\section{El modelo didáctico de investigación en Iberoamérica}

De acuerdo con Gil Pérez y Valdés Castro (1996), es necesaria una reorientación de las prácticas de laboratorio "para que dejen de ser meras ilustraciones de los conocimientos transmitidos y pasen a constituir actividades de investigación» (p. 155). Este replanteamiento constructivista de las prácticas educativas intenta reformular la educación de ciencias más allá de los modelos de cambio conceptual que hasta la década de los ochenta dominaban la enseñanza de las ciencias (Gil Pérez, 1994; Moya y Campanario, 1999). En este sentido, la práctica como investigación dirigida implica más que solo experimentación, pues requiere la integración de otros aspectos de la actividad científica auténtica (Gil Pérez y Valdés Castro, 1996), tales como las situaciones problemáticas abiertas, la relevancia de las situaciones propuestas, los análisis cualitativos y la planificación de la actividad experimental por los propios estudiantes, entre otros (Carrascosa et al., 2008).

\section{El modelo didáctico de investigación en el mundo anglosajón}

De acuerdo con el reporte del National Reseach Council (National Research Council. Center for Science, 2000), los estándares en Estados Unidos enfatizan la enseñanza de la investigación como parte central de la educación científica. Posterior a la década de 1960 se hizo énfasis en que las prácticas experimentales y el trabajo de laboratorio debían preceder la explicación formal de los conceptos y principios científicos. No obstante, actualmente el énfasis no es sólo acerca de la enseñanza de la práctica de investigación, sino también la enseñanza de cómo ésta desemboca en la edificación del conocimiento científico. Así pues, la educación en Estados Unidos ha planteado el objetivo adicional de enseñar a los estudiantes las concepciones de la naturaleza de la ciencia (Lederman, 1992). Con todo, la enseñanza de la práctica de investigación en la enseñanza de las ciencias es también uno de los temas centrales de los currículos en el contexto internacional (Abd-El-Khalick et al., 2004).

\section{El modelo didáctico de investigación en Colombia}

Aunque se han delineado varias perspectivas didácticas en la historia de la enseñanza de las ciencias en Colombia (por ejemplo, C. A. Hernández, 2001), aún parece evidente la falta de una práctica didáctica en donde la experimentación sea habitual (Oliva-Martínez y Acevedo-Díaz, 2005). En efecto, nuestra situación educativa históricamente no se ha caracterizado por prácticas didácticas basadas en la experimentación (Ortega, 2007), y pareciera que la tendencia de una enseñanza tradicional aún sea dominante en los salones de clase. Igual que en el contexto anglosajón, también en el contexto iberoamericano se ha propuesto la necesidad de actualizar las prácticas educativas e incluir la enseñanza de la naturaleza de las ciencias de la mano de la experimentación (Acevedo Díaz, 2008). Asimismo, también en Colombia se ha enfatizado la necesidad de incluir actividades auténticas de investigación 
como parte de la enseñanza escolar. Por ejemplo, Hernández y otros (2004) afirman que «la forma en que los científicos descubren e interpretan los fenómenos del mundo, es posiblemente la estrategia más significativa y adecuada posible para aprender ciencias naturales.» (pág. 52).

A continuación se presenta el contexto de la educación en la sociedad de la información. Se intenta mostrar cómo, en esta época permeada por las TIC, la educación debe hacer frente a la alfabetización en nuevas herramientas tecnológicas y responder a nuevas formas no sólo de entender el mundo, sino de aprender en la escuela. Así pues, se pretende mostrar cómo ésta nueva forma de aprender requiere de una práctica escolar basada en la experimentación.

\section{Necesidad de implementación de nuevas herramientas pedagógicas}

Nuestro conocimiento científico sobre los fenómenos del mundo proviene de registros mediados por instrumentos tecnológicos de muchas clases. Estos instrumentos le permiten al científico ir más allá de sus limitaciones perceptuales, y se encuentran estrechamente relacionados con la comprensión del fenómeno estudiado. Esta tendencia tiende a aumentar en la ciencia del siglo XXI (Kozma, 2000).

Como bien lo prescriben D. Thomas y J.S. Brown (2011), la agencia del conocimiento en la nueva cultura de aprendizaje la ejerce el estudiante en su interacción con los recursos tecnológicos que hoy en día se hallan a su disposición. En la educación de nuestro siglo, siendo nosotros los educadores, deberíamos entender cómo aprovechar los nuevos recursos que existen a nuestro alcance. Con estos recursos, el estudiante puede jugar, cuestionar e imaginar, y de esta manera ingresar en esta nueva cultura de aprendizaje.

Varios autores han planteado la existencia de lo que se ha llamado los «principios tecnológicos de automatización de experimentos». Según Valdés Castro y Valdés Castro (1994), los computadores plantean nuevos objetivos y contenidos en la enseñanza de las ciencias. Entre otros, estos autores proponen formar conocimientos y habilidades para la experimentación con modelos creados en el computador, e introducir a los estudiantes en los principios tecnológicos de la automatización de experimentos. (Un ejemplo de aplicación de estos principios en la Educación Secundaria acerca de la caída de los cuerpos se encuentra en Gil Pérez y Valdés Castro, 1996; y un ejemplo de aplicación en educación universitaria y empleando también un ambiente b-learning se puede consultar en Ferrini y Aveleira, 2006).

\section{La fotografía digital en la práctica educativa de las ciencias}

En su investigación sobre la educación digital, Battro y Denham (1997) predijeron que los nuevos instrumentos del pensamiento humano transformarán la educación en las próximas generaciones. Entre otros instrumentos tecnológicos que describen, como Internet, el correo electrónico, el escáner, los procesadores de texto, etc., estos autores mencionan la cámara fotográfica, la videograbadora y otros sistemas multimedia. Sobre estos mencionan que antes "Cada profesional tenía a su cargo un equipo, computadora, cámara fotográfica, videograbadora, etc. Compaginar las diferentes producciones era una tarea de titanes. Hoy día el proceso se ha simplificado de tal manera que la producción de un multimedio se convertirá muy pronto en una tarea escolar más, en manos de los propios alumnos» (p. 108, el énfasis es mío). En particular, estos autores mencionan a la cámara digital, la cual permite utilizar las fotos al breve instante luego de tomarlas y posteriormente almacenarlas en un computador. En los proyectos de clases, el alumnado puede usar estas cámaras en sus excursiones y trabajos de investigación. Con respecto a la videograbadora, mencionan que esta será usada en proyectos artísticos, presentaciones y videoconferencias. 
En efecto, la cámara digital en manos de los estudiantes se presenta como una realidad de nuestra época. Aguaded Gómez y Sánchez Carrero (2008) argumentan que esta situación, colocar a «los niños tras el visor de la cámara», es una cuestión de alfabetización infantil y juvenil. Cada vez es más barato y fácil acceder a estos recursos audiovisuales como herramientas didácticas dentro del salón de clase.

En un ejemplo de aplicación de la fotografía como apoyo a la experimentación científica, presentado en Gil Pérez y Valdés Castro (1996), y también en Carrascosa y otros (2008), se menciona cómo la toma de fotos se utiliza para estudiar la aceleración uniforme en la caída de los cuerpos. Dado que los tiempos de caída de los objetos son tan pequeños, no es posible realizar mediciones exactas y útiles basándose únicamente en la percepción sensorial de la vista. En la investigación citada se menciona como los estudiantes proponen fotografiar la caída para estudiar el movimiento de los cuerpos:

(Los estudiantes) conciben entonces la posibilidad de fotografiar la caída de la bola, al lado de una cinta métrica, con una cámara que dispare automáticamente a intervalos de tiempo regulares y muy breves. Este procedimiento de fotografía estroboscópica ha sido utilizado en este tipo de medidas con muy buenos resultados y así conviene hacérselo notar a los estudiantes a modo de refuerzo de sus planteamientos. La idea de la automatización aparece como algo básico para evitar los problemas de coordinación entre el instante de soltar la bola y la puesta en marcha del cronómetro. En ese sentido surge también la propuesta de utilizar relojes electrónicos, que se pongan en marcha al soltarse la bola y se paren al chocar esta contra un tope (Carrascosa, 2006, p. 171).

\section{TRES PROPUESTAS DIDÁCTICAS PARA LA EDUCACIÓN CON LA CÁMARA DIGITAL}

A continuación se presentan tres estudios de caso de propuestas didácticas que intentan abordar la problemática elaborada en este artículo, es decir, la educación de una generación digital con herramientas didácticas basadas en la cámara digital. Estas propuestas han sido validadas con profesoras en formación de una licenciatura en educación para la primera infancia de una universidad privada en la capital de nuestro país. Las tres experiencias que se narran a continuación tuvieron lugar durante los cuatro semestres académicos comprendidos entre el segundo semestre de 2009 y el primero de 2011. En estas experiencias participaron el investigador principal, que era el docente encargado de la asignatura Enseñanza de las Ciencias en ese periodo, y un grupo de estudiantes de la licenciatura cada semestre. Las estudiantes cursaban su tercer año de estudios y en cada grupo participaron aproximadamente diez alumnas. Los ejemplos de evidencias que aquí se presentan provienen de los proyectos que realizaron durante el semestre. En el tercer caso, llamado «El taller de fotografía para alumnado de preescolar», las estudiantes realizaron este taller con niños mientras ellas asistían a sus prácticas académicas en varios colegios de la ciudad.

\section{Estudio de caso 1: la caída de los cuerpos}

Un preconcepto muy arraigado en el pensamiento físico de la gente es que los cuerpos más pesados caen más rápido que los objetos más livianos. Aunque esto puede parecer cierto para una hoja de papel, la misma hoja arrugada y doblada hasta formar una pequeña bola cae a la misma velocidad que un llavero. Cabe anotar que la bola de papel pesa igual que cuando tenía la forma de una hoja plana (en ocasiones hemos recibido comentarios por parte de algún estudiante que piensa que la conservación del peso no se mantiene). Pero ¿cómo estar seguro de que estos cuerpos, la bola de papel y el llavero, caen al mismo tiempo? 
Esta cuestión no es fácil de resolver, y su comprobación empírica en el salón de clase y a ojos vista está llena de errores de medición que pueden falsear el resultado. Usualmente, tal comprobación empírica tiene un efecto interesante entre los estudiantes que hacen de público, y quienes han puesto a prueba sus hipótesis: ¿cuál caerá primero? No obstante, no falta quien aún no queda convencido, incluso luego de haber observado que caen simultáneamente ambos, bola de papel y llavero.

Una actividad interesante, llamativa, y que suscita la participación de los estudiantes, es el uso de una cámara digital y varios materiales que se consiguen fácilmente: una pizarra, una serie de objetos de variado tamaño y peso, y una báscula portátil. En pequeños grupos, la actividad se inicia recordando los pasos de una investigación, y con la división del trabajo dentro de los grupos. Los estudiantes pesan uno a uno los objetos, supongamos que son tres. Luego, un estudiante toma un objeto y se para frente a la pizarra. Se marca en esta un punto de partida y uno de llegada al estilo de una cinta métrica (se puede usar una de éstas si se dispone de ella).

Luego, el estudiante suelta el objeto desde la marca de salida mientras otro graba en video con la cámara digital (ilustración 1). Otro estudiante es el encargado de transferir el video a un computador portátil. Posteriormente, se construye y se lleva un registro en una hoja de cálculo con una tabla con los pesos respectivos de los objetos. También se incluye el tiempo exacto en que cada objeto pasa la raya de salida y luego el tiempo exacto en que pasa la raya de llegada. Esto es posible porque cualquier programa de edición de video (que por defecto se encuentra instalado en la mayoría de sistemas operativos) muestra la película cuadro a cuadro, llegando incluso a una velocidad de un cuadro por cada 0,04 segundos. Así pues, el margen de error se reduce considerablemente, lo que permite calcular el tiempo exacto que gasta cada cuerpo en pasar la raya de salida hasta pasar la raya de llegada. El tiempo que toma cada objeto es la resta del momento de llegada menos el momento de salida.

Para el asombro de muchos, esta última columna de la tabla en la hoja de cálculo muestra valores muy similares (tabla 1). Posteriormente, los estudiantes completan el ciclo de investigación, reflexionando y escribiendo un reporte final con sus conclusiones. Paralelamente, en una discusión con toda la clase, todos los grupos comparan sus resultados entre sí, así como sus dificultades metodológicas y sus ingeniosas maneras de solucionarlas. Por último, es valioso comentar que las estudiantes que participaron en este proyecto realizaron un ensayo escrito reflexionando acerca de cómo esta experiencia puede ser útil para su práctica educativa. Entre algunas ideas discutidas, las estudiantes destacaron la función del método científico y la importancia de recolectar evidencia empírica para sustentar las teorías científicas.

Tabla 1.

Hoja de cálculo con la computación del tiempo total de caída de varios objetos

\begin{tabular}{|l|c|c|c|}
\multicolumn{1}{c}{ Objeto } & \multicolumn{1}{c}{ t suelta } & t llega & t caída \\
\hline billetera & 0,07 & 0,43 & 0,36 \\
\hline cartuchera & 0,37 & 0,73 & 0,36 \\
\hline marcador & 0,86 & 1,22 & 0,36 \\
\hline
\end{tabular}

Hace cientos de años, Galileo Galilei usó una clepsidra y las pulsaciones del corazón como ayuda para medir el tiempo de caída. Incluso ingenió el uso de un plano inclinado para reducir la velocidad de caída de los objetos. Si Galileo hubiera tenido una cámara digital, el ser humano no hubiera tenido que esperar hasta llegar a la Luna para comprobar empíricamente sus ideas. Pero más allá de ello, la ciencia se fortaleció con el poder de las inferencias de este genio italiano. Es aquí donde el rol de las docentes debe guiar la discusión y darle valor agregado a la reflexión del aprendizaje por investigación. 

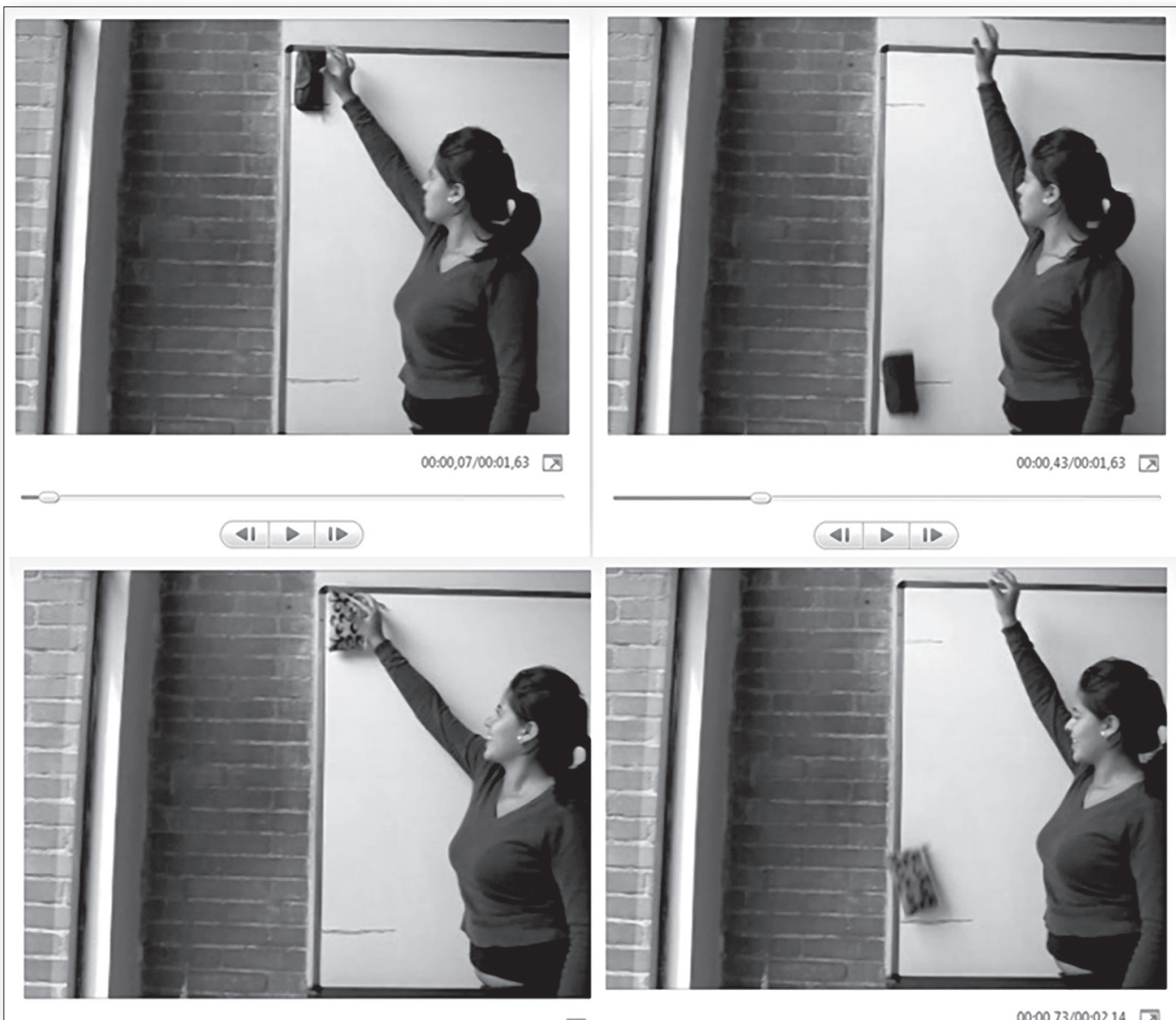

$00: 00,37 / 00: 02,14 \pi$

$00: 00,73 / 00: 02,14 \pi$
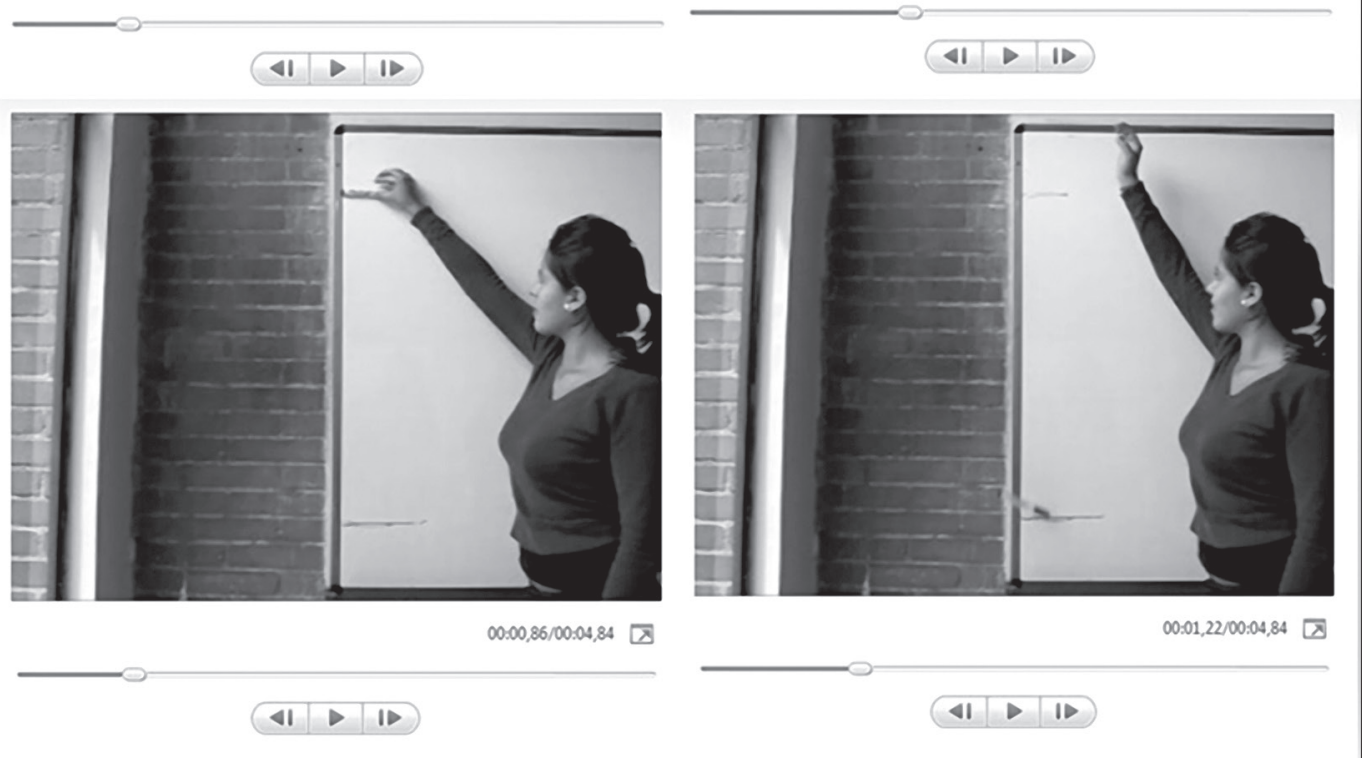

$$
41 \triangleright \mid 1 \nabla
$$

$00: 00,86 / 00: 04,84 \pi$

Ilustración 1. Una muestra de cuadros del video obtenido en la experimentación de la caída de varios objetos. Proyecto realizado por cuatro estudiantes de la licenciatura de Educación en Primera Infancia durante el primer semestre de 2011. 


\section{Estudio de caso 2: la inclinación del eje de la Tierra y su recorrido alrededor del Sol}

¿Quién se ha detenido a prestar atención al movimiento del Sol? Esta es una pregunta con la que usualmente comienzo la clase. La observación científica implica un registro sistemático, prolongado y meticuloso. Solo así puede uno comenzar a percatarse de los cambios y regularidades en todo aquello que uno da por sentado, tal como el hecho de que el Sol sale y se pone todos los días.

El Sol sale por el este y se pone por el oeste (desafortunadamente este hecho convencional no lo conocen todos los estudiantes). En la mañana el Sol asciende hasta llegar a su parte más alta al medio día, y luego desciende hasta ocultarse al final de la tarde. Se les ha pedido a los estudiantes que tomen una foto al Sol desde la ventana de su casa, temprano en la mańana o al final de la tarde, todos los lunes de cada semana. La condición necesaria es que se tome la foto desde la misma parte cada vez, en la misma posición y a la misma hora. Es decir, es necesario un punto de referencia que aparezca fijo en todas las fotos, así como anotar la fecha en que se toma cada foto. Muchos estudiantes se quedan esperando que sea una broma, pues no parece una tarea muy entretenida. Puede que no lo sea, pero no toma más de cinco segundos cada vez.

Realizando esta tarea durante un periodo de tiempo suficientemente largo, cuatro meses por ejemplo, la paciencia comienza a dar sus frutos. Las fotos muestran que la posición del Sol no es exactamente igual en cada una de las fotografías, pues esta ha venido cambiando con el tiempo y con relación al punto que se tomó como referencia. En efecto, en las fotos que realizó una de mis estudiantes, por ejemplo, el Sol que antes estaba hacia la derecha de la foto, ahora aparece hacia la izquierda y más abajo (ilustración 2). Puede apreciarse en la ilustración 2 que las tres fotos tienen el mismo punto de referencia: es posible ver dos postes de electricidad, uno en cada esquina inferior de la foto; además, cada foto lleva la fecha en que fue tomada, 8 de julio, 18 de agosto y 26 de octubre. Por supuesto, la dirección del movimiento del Sol variará dependiendo de si las fotos son tomadas por la mañana, por la tarde, de enero a junio o de julio a diciembre, en el hemisferio sur o norte, en fin, de dónde y cuándo se realicen. Lo cierto es que, de cualquier manera, el Sol parece moverse con el paso de los meses.

El objetivo del taller es realizar un registro sistemático y fenomenológico, un punto de partida básico en experimentación. Con ayuda de las fotos digitales, el observador tiene la posibilidad de visualizar el desplazamiento del Sol. Este registro sistemático tiene varias características: $a$ ) es un registro continuado a lo largo de un periodo de tiempo; $b$ ) la observación y el registro requieren un punto de referencia, cambiante según el observador; $c$ ) el registro permite la marcación de puntos de desplazamiento en correspondencia con los intervalos de tiempo de las fechas en que fueron tomadas las fotos; y, finalmente, d) la fotografía digital permite trazar fácilmente las coordenadas del desplazamiento en el espacio. En efecto, es posible medir el movimiento aparente del Sol con ayuda del registro realizado con la cámara digital. Llamando $X$ a la distancia horizontal e $Y$ a la distancia vertical, es posible referirse a cuánto ha cambiado la posición del Sol con el pasar de los meses del año (ilustración 3). Ahora bien, cuando el experimento del registro del movimiento del Sol se repite semestre a semestre, se hace notar que el desplazamiento es igual durante el primer semestre del ańo, y que tal movimiento se invierte durante el segundo semestre.

Pero ¿por qué se ha desplazado el Sol? ¿Se mueve el Sol, o se mueve la Tierra? Estas preguntas no son fáciles de contestar, y su respuesta requerirá el uso de un modelo concreto, gráfico o imaginario, del sistema Solar. No obstante, esta búsqueda cae por fuera del objetivo del presente taller de observación fenomenológica con la cámara digital, y requerirá ser tratado en otro momento, incluso podría esperar a ser tratado en otro año escolar. Son varios factores que simultáneamente es necesario conceptualizar, a saber: la órbita elíptica de la Tierra alrededor del Sol, con este último en uno de los focos de la elipse, los movimientos de traslación y rotación, y la inclinación de 23 grados del eje de rotación de la Tierra. 


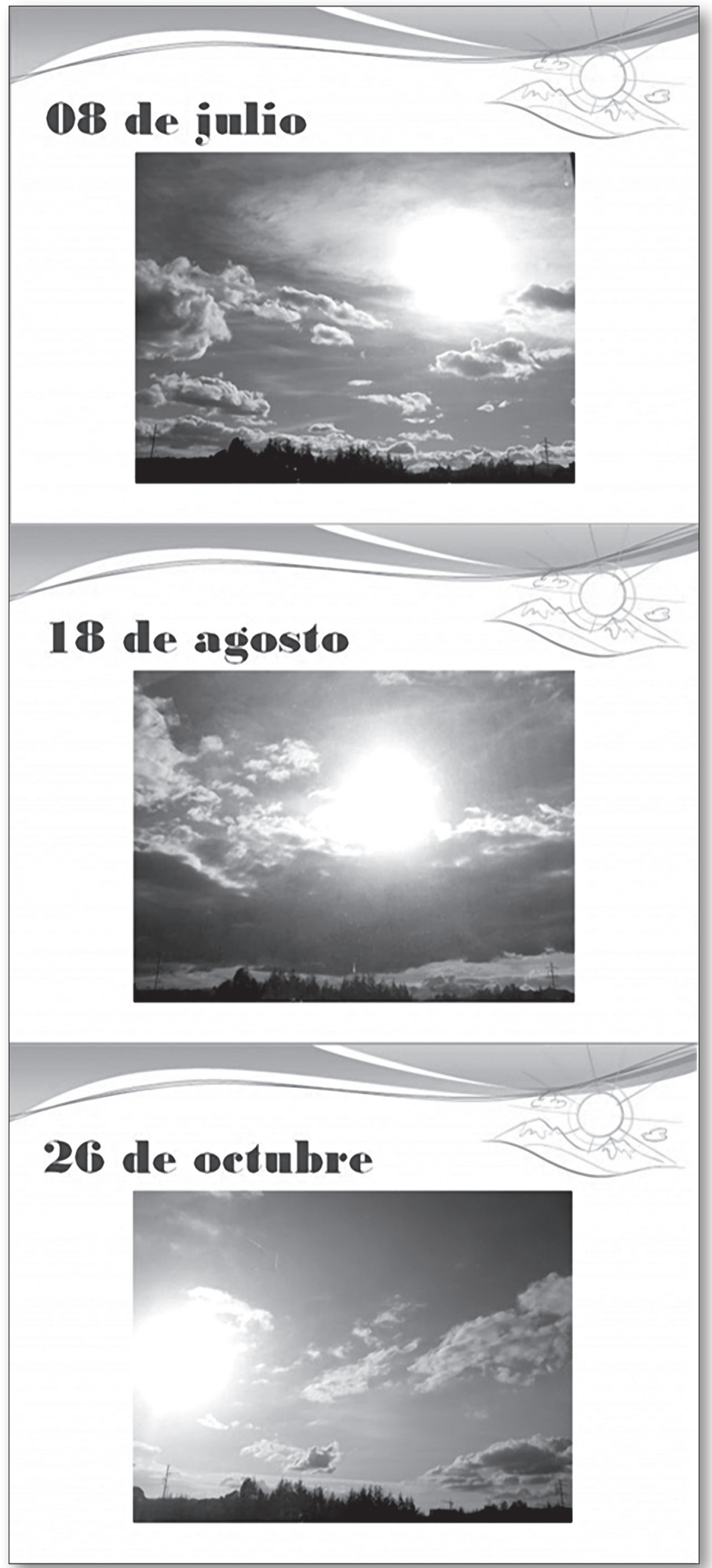

Ilustración 2. Observación del movimiento aparente del Sol en el firmamento realizada durante cuatro meses por una estudiante de la licenciatura de Educación en Primera Infancia durante el segundo semestre de 2010. 


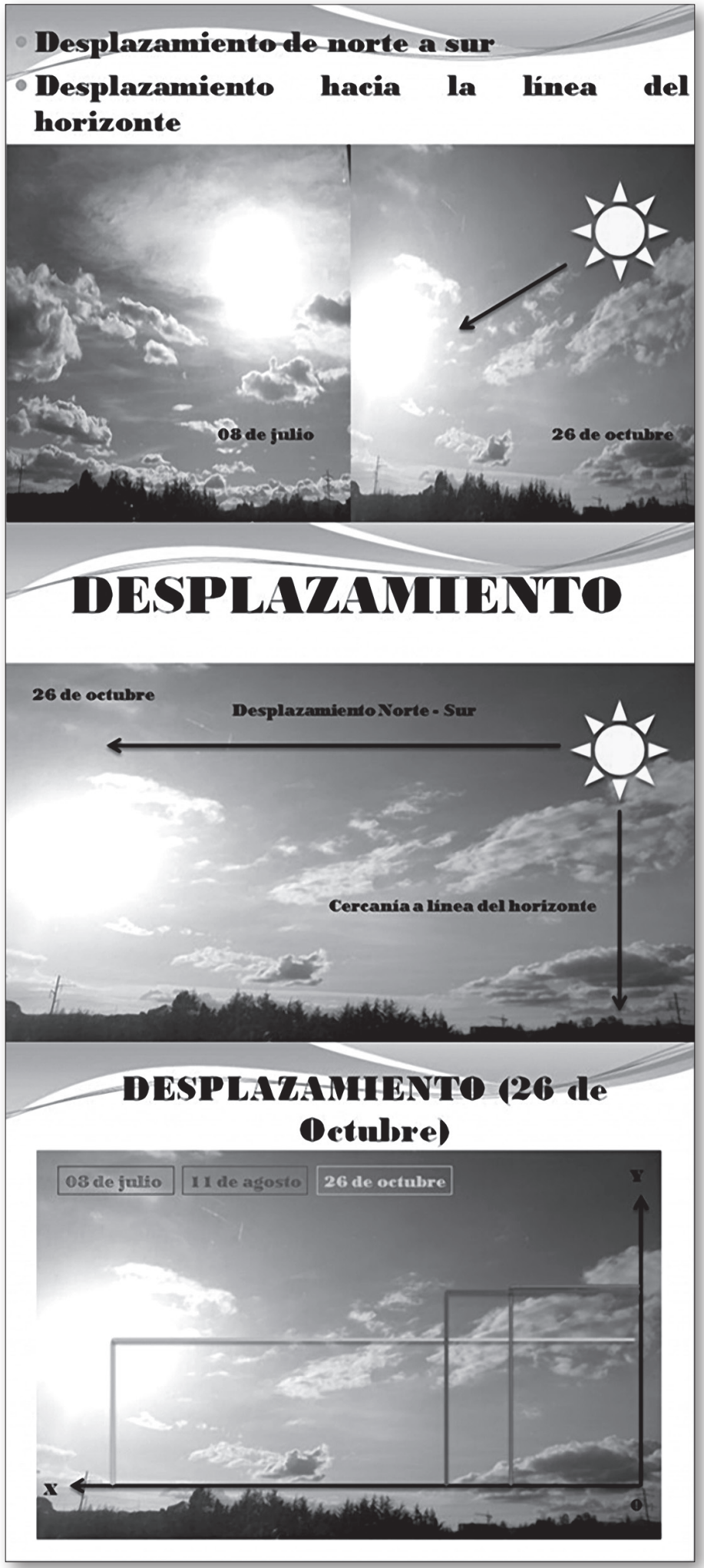

Ilustración 3. Ejemplo de la medición del movimiento aparente del Sol. Estas imágenes forman parte de la presentación del proyecto final de la misma estudiante 
Nuestros antepasados estaban enfrentados a explicar este tipo de observaciones metódicas, sostenidas y meticulosas. Ellos fueron responsables de desvelar los cambios de los fenómenos físicos, pero también las regularidades de esos cambios, lo que llevó a construir explicaciones en forma de modelos. Además, «a través de la astronomía, de la astrología o de la cosmología, la observación de los cielos ha sido, sin duda, la mayor influencia en el desarrollo de las matemáticas.» (Mankiewicz, 2005: 20). En la mente del observador se intenta construir sentido y explicar un fenómeno que así lo requiere. Es tarea de la profesora, en un segundo taller más avanzado, organizar una exposición guiada acerca del modelo del sistema Solar, incluso ilustrada o recreada en una animación computarizada, y tratar de encaminar el pensamiento de los estudiantes hacia esta manera compleja y elaborada de entender el fenómeno.

No obstante, desafortunadamente se ha mostrado que el preconcepto es muy fuerte, y el modelo del sistema solar requiere mucha abstracción, así como descentrar el pensamiento, es decir, ponerse en el lugar de un observador imaginario. Incluso se ha comentado que estudiantes de universidad encuentran difícil explicar cómo se originan las estaciones, y recurren a ingeniosas explicaciones (Lehrer y Schauble, 2006). Podemos comentar que pudimos evidenciar algo parecido con varios de nuestros estudiantes a lo largo de las experiencias con el proyecto de observación del Sol. En general, el paso conceptual desde la observación y el registro hacia la construcción del modelo presentaron mucha resistencia por parte del alumnado.

No obstante, la semilla está sembrada para mostrar que el avance de la ciencia requiere plantearse problemas de interés, elaborar hipótesis, observar, medir, construir modelos, teorías y discutirlas en el seno de la comunidad científica (Latour, 1987). Adicionalmente, varios autores (Latour, 1990; Lynch, 1988; Roth y McGinn, 1998) han señalado cómo los registros materiales generados por la investigación científica, en este caso con la cámara digital, son una parte fundamental del trabajo científico. Meira (1995), refiriéndose al rol de las representaciones materiales en la actividad científica y citando a Latour, dice: «En este sentido, los científicos nunca han medido al Sol, sino solo fotografías de este.» (p. 272).

\section{Estudio de caso 3: el taller de fotografía para alumnado de prescolar}

Basándonos en el artículo de Byrnes y Wasik (2009) acerca del uso de la fotografía como una herramienta de aprendizaje en el salón de preescolar, preparamos una adaptación para realizar un taller de fotografía en el que participaran nińos de edad preescolar. En resumen, la actividad consistió en dos momentos, una salida de campo y una en el salón de clases. En la salida de campo, orientada por profesoras en formación, cada preescolar llevaría una cámara digital (idealmente). Durante la salida, se toman fotografías de lo que les llamó la atención. Si no es posible una salida de campo, ellos o ellas pueden tomar fotos de objetos de su entorno cercano, de su casa, su familia, del parque donde juegan, etc. Lo importante es que sean los preescolares quienes tomen las fotos, y por ello que capturen aquello que sea de su interés. Y de esta manera, en la actividad, cuando regresen al salón de clase, que la dinámica de trabajo provenga del propio interés del alumnado, que se produzca de una manera natural.

En esta segunda actividad, la cual se puede realizar en el salón de clase, las fotos se montan en una presentación de Power Point y una a una estas se proyectan sobre una pantalla mientras se hacen preguntas a los preescolares (ilustración 4). Lo más importante aquí es que las preguntas y la retroalimentación realizadas por la profesora cumplan dos objetivos: el primero es activar el conocimiento previo; el segundo es acercar a estos niños y nińas al mundo social y cultural de los adultos.

Las preguntas iniciales que se hacen indagan qué tanto saben los preescolares acerca del objeto, animal o persona que aparece en la foto que hayan tomado. Las preguntas pueden ser muy generales, pero en cualquier caso hay que obviar la pregunta: «¿Dime, Juanita, por qué tomaste esta foto?». En general, si Juanita tomó esa foto fue porque le gustó, le pareció interesante y llamativo el objeto de 
la foto (y esa era precisamente la intención de darle la cámara a ella). Al contrario, el orientador del taller debe prestar mucha atención a esta instrucción antes de efectuar las preguntas, pues encontré varios casos en los que las orientadoras solo preguntaron eso. En general, a esta pregunta el preescolar se limita comúnmente a responder, por ejemplo: «Porque me pareció bonito».

Las preguntas que suscitan una interesante dinámica de diálogo son aquellas que indagan en el conocimiento previo del alumnado. Por ejemplo, en la tabla 2 el lector puede encontrar sugerencias para realizar preguntas acerca de animales, objetos o personas.

Tabla 2.

Ejemplos de preguntas que suscitan una discusión constructiva y activan el conocimiento previo del alumnado

\begin{tabular}{|c|c|c|}
\hline ANIMALES & OBJETOS & PERSONAS \\
\hline $\begin{array}{l}\text { Si se tomaron fotos a una paloma, se } \\
\text { puede preguntar: }\end{array}$ & $\begin{array}{l}\text { Si es un semáforo, por ejemplo, se } \\
\text { puede preguntar: }\end{array}$ & $\begin{array}{l}\text { Si las fotos son de una persona, se pue- } \\
\text { de preguntar: }\end{array}$ \\
\hline $\begin{array}{l}\text { - ¿Sabes qué es eso? } \\
\text { - ¿Sabes cómo se llama este pájaro? } \\
\text { - ¿Y dónde vive? } \\
\text { - ¿Y de qué se alimenta? } \\
\text { - ¿Y tiene plumas o pelo? } \\
\text { - ¿Y dónde nace? } \\
\text { - ¿Y qué otro tipo de paloma has visto } \\
\text { o conoces? }\end{array}$ & $\begin{array}{l}\text { - ¿Cómo se llama esto? } \\
\text { - ¿Dónde lo viste? } \\
\text { - ¿Dónde más lo has visto? } \\
\text { - ¿Y sabes para qué sirve? } \\
\text { - ¿Y cómo funciona? } \\
\text { - ¿Y es importante para la gente? } \\
\text { - ¿Qué pasa si no está funcionando? } \\
\text { - ¿Qué otro tipo de semáforos has } \\
\text { visto, por ejemplo en otras ciudades? } \\
\text { - ¿Y la señal de «Pare» funciona igual? }\end{array}$ & $\begin{array}{l}\text { - ¿Cómo se llama esta persona? } \\
\text { - ¿De quién es hijo/a? } \\
\text { - ¿Y qué relación de parentesco tiene } \\
\text { contigo? } \\
\text { - ¿Quién es su hermano/a? } \\
\text { - ¿Qué edad tiene? } \\
\text { - ¿Qué diferencia de edad hay entre } \\
\text { vosotros? } \\
\text { - ¿Conoces a qué se dedica? }\end{array}$ \\
\hline
\end{tabular}




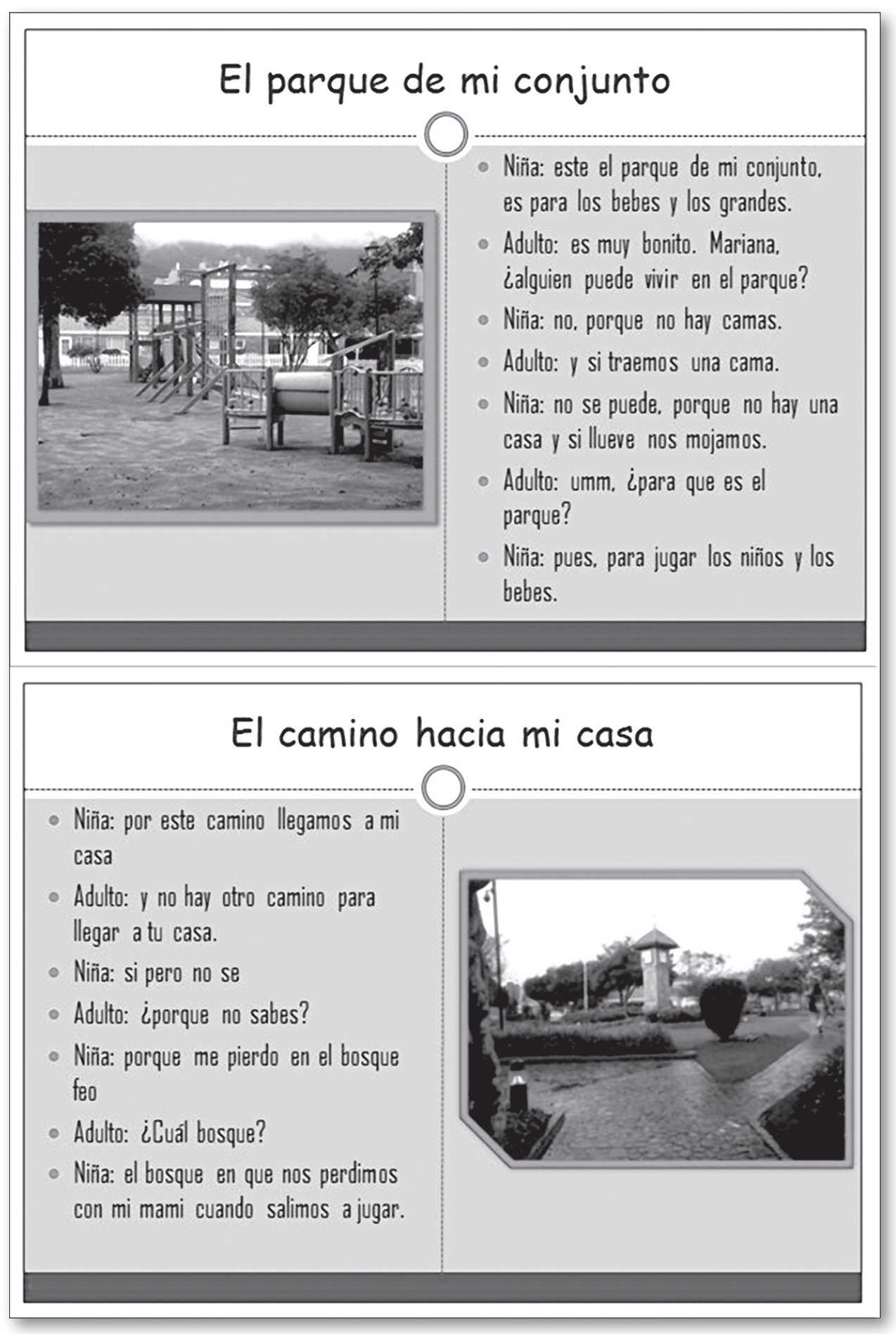

Ilustración 4. Taller de fotografía con alumnado de prescolar. Actividad realizada por dos estudiantes de la licenciatura y un niño de 5 ańos durante el primer semestre de 2011 
Para hacer preguntas realmente constructivas se requiere que la profesora en formación observe previamente las fotografías que ha tomado cada niño o niña, y pueda preparar el tipo de preguntas que deberá utilizar para orientar correctamente la conversación. Aproximadamente cada pequeño debe tomar entre cinco y diez fotos.

Adicionalmente, también se pueden realizar otras actividades colectivas con la cámara fotográfica, como juegos y actividades de clase. Por ejemplo, cada estudiante toma una foto en el salón de clase sin que los demás se percaten de dónde o a qué le ha tomado una foto. Luego, la profesora proyecta las fotos una por una y en cada ocasión los niños tienen que buscar el objeto o el lugar correspondiente a la imagen que se proyecta. También se describen otras actividades interesantes que se pueden encontrar en el artículo de Byrnes y Wasik (2009) y que cualquier docente puede ensayar para que sus estudiantes las realicen. También se pueden encontrar otras actividades para la enseñanza de idiomas en preescolar usando la cámara digital y la comunicación visual en el artículo de Britsch (2010) acerca del uso de folletos de fotos para el aprendizaje del inglés.

En fin, lo importante es, como afirma Trujillo de Figarella (2001), conseguir el desarrollo de la actitud científica en alumnado de edad preescolar. Para ello, se debe aprovechar la curiosidad innata, constante y despierta de ellos, así como su necesidad de explicar y encontrar respuestas a problemas de su vida cotidiana.

\section{DISCUSIÓN}

Construir una actitud científica y de investigación por parte de los estudiantes requiere un cambio también en la actitud de los docentes. Yoo (2010) ha argumentado que los esfuerzos por mejorar la formación profesional de los docentes en ciencias pueden haber fallado debido a no haberse considerado la necesidad de fomentar un cambio en la actitud y el pensamiento reflexivo de los docentes. En efecto, la disposición y actitud del docente son factores cruciales en el salón de clase que influencian el éxito de la educación científica. En prácticas con el uso de la cámara digital como herramienta didáctica es igualmente importante la actitud del docente.

Adicionalmente, Hammer (1997) ha propuesto no solo la existencia de un aprendizaje por descubrimiento, sino también una «enseñanza por descubrimiento». Según él, el profesorado interesado en promover la enseñanza por investigación frecuentemente siente la tensión entre dedicar tiempo a la experimentación y la necesidad de cubrir contenido. Además, en la enseñanza por experimentación los estudiantes pueden llegar a «descubrimientos» que no estaban previstos en el currículo. La instrucción exitosa dependerá de la comprensión y las percepciones no anticipadas que pueda hacer la docente, es decir, ser hábil en lo que él ha llamado la enseñanza por descubrimiento. En este sentido, la investigación científica apoyada por la cámara digital requiere del manejo de nuevas expectativas por parte del profesorado acerca de la enseńanza en el salón de clase. No solo se ponen en juego las competencias investigativas de cada estudiante, sino que las imágenes y los videos capturados por ellos facilitan al docente visualizar la manera como ellos están comprendiendo el fenómeno, así como también sirven de apoyo para la discusión y la colaboración en grupo.

Junto a la actitud y las expectativas del docente, también es necesario considerar las prácticas epistémicas de los estudiantes en su educación en investigación científica (Duschl, 2008; Sandoval y Reiser, 2004). Según Sandoval (2005), no es suficiente con enseñar en la escuela los aspectos epistemológicos formales de la ciencia, es decir, la naturaleza de la ciencia, pues poco se sabe acerca de las creencias epistemológicas que guían la investigación de los estudiantes. Es decir, se hace necesario comprender las prácticas epistemológicas que guían la actividad de los estudiantes al realizar la investigación en el salón de clase. En otras palabras, las ideas que los estudiantes tienen acerca de sí mismos como apren- 
dices científicos influyen más en sus esfuerzos por aprender la ciencia que aquellas ideas de una ciencia formalizada y distante. Por ello, lo importante entonces es enfocar el estudio de las prácticas por medio de las cuales el alumnado construya su conocimiento. De esta manera, en nuestra investigación mostramos cómo el uso de la cámara digital puede apoyar las prácticas epistémicas de los aprendices de ciencias. Las imágenes obtenidas en los experimentos y en la observación naturalista aportan los recursos para que el o la estudiante pueda sistematizar fácilmente el registro fenomenológico por medio del cual expresa su comprensión. Estos recursos visuales pueden ser a su vez organizados e integrados con otras representaciones científicas, como las gráficas y tablas que con frecuencia son usadas en la práctica científica auténtica (Friel, Curcio y Bright, 2001; Roth y McGinn, 1997).

En resumen, el uso de la cámara digital como herramienta didáctica apoya diversos e importantes procesos educativos que responden a una perspectiva constructivista de la enseñanza de las ciencias. Sin embargo, es indispensable acompañar esta herramienta de nuevas prácticas educativas y de aprendizaje por parte de los estudiantes. Estos cambios deben ir acompañados de actitudes, disposiciones y expectativas congruentes con el aprendizaje basado en la experimentación, el descubrimiento y la reflexión.

\section{CONCLUSIONES}

El objetivo del presente artículo ha sido mostrar cómo la cámara digital es una herramienta didáctica para transformar la práctica de la educación en ciencias en nuestros salones de clase en el nivel de educación básica. Para ello, aquí hemos presentado tres casos de proyectos de clase que tienen en común el uso de la cámara digital. Estos casos responden a una teoría de la enseñanza de las ciencias y a una nueva cultura de aprendizaje basadas en la fundamentación de las prácticas de investigación, cuya principal herramienta es, por supuesto, la experimentación. En efecto, los tres ejemplos comparten la misma mirada constructivista de la educación basada en el modelo por investigación. El primer caso presentó el estudio de la caída de los cuerpos; el segundo caso la observación del Sol como un cuerpo celeste, y el tercer caso el uso de la cámara digital por estudiantes de primera infancia.

Esperamos que los casos ilustrativos aquí presentados puedan aportar a los docentes en Educación Primaria y Secundaria ejemplos concretos para su propia práctica. De esta manera se podrá contribuir con la transferencia a la práctica educativa de las ideas presentadas en esta investigación. Además, esperamos que la lectura de estas propuestas motiven a otros investigadores y estudiosos de la didáctica específica de las ciencias a presentar sus propias ideas. Así, será posible desarrollar una comunidad de investigación en torno a la enseñanza de las ciencias en el contexto de las nuevas tecnologías de la información. Aún son necesarias futuras investigaciones y aplicaciones de didácticas específicas y concretas en el salón de clase. El objetivo será continuar arrojando luz a nuevas maneras de educar a las generaciones que crecen en una sociedad digital y que demandan una nueva cultura de aprendizaje.

\section{AGRADECIMIENTOS}

Estoy en deuda con la buena voluntad y el esfuerzo que mis estudiantes pusieron en mis cursos, así como con su paciencia y persistencia para elaborar los productos de los que aquí solo se incluye una pequeña muestra. 


\section{REFERENCIAS BIBLIOGRÁFICAS}

Abd-El-Khalick, F., BouJaoude, S., Duschl, R., Lederman, N. G., Mamlok-NaAman, R., HofsteIn, A. y TuAn, H. (2004). Inquiry in science education: International perspectives. Science Education, 88(3), pp. 397-419.

http://dx.doi.org/10.1002/sce.10118

Acevedo Díaz, J. A. (2008). El estado actual de la naturaleza de la ciencia en la didáctica de las ciencias. Revista Eureka sobre Enseñanza y Divulgación de las Ciencias, 5(2), pp. 134-169.

Aguaded Gómez, J. I. y Sánchez Carrero, J. (2008). Niños y adolescentes tras el visor de la cámara: experiencias de alfabetización audiovisual. Estudios sobre el mensaje periodístico (14), pp. 293-308.

Battro, A. M. y Denham, P. J. (1997). La educación digital una nueva era del conocimiento. Buenos Aires: EMECE.

Britsch, S. (2010). Photo-Booklets for English Language Learning: Incorporating Visual Communication into Early Childhood Teacher Preparation. Early Childhood Education Journal, 38(3), pp. 171-177. http://dx.doi.org/10.1007/s10643-010-0412-2

Byrnes, J. y Wasik, B.A. (2009). Picture This: Using Photography as a Learning Tool in Early Childhood Classrooms. Childhood Education, 85(4), pp. 243-249. http://dx.doi.org/10.1080/00094056.2009.10523090

Carrascosa, J., Pérez, D.G., Vilches, A. y Valdez, P. (2008). Papel de la actividad experimental en la educación científica. Caderno Brasileiro de Ensino de Física, 23(2), pp. 157-181.

Duschl, R. (2008). Science education in three-part harmony: Balancing conceptual, epistemic, and social learning goals. Review of Research in Education, 32(1), pp. 268-291. http://dx.doi.org/10.3102/0091732X07309371

Edelson, D.C., Gordin, D.N. y Pea, R.D. (1999). Addressing the Challenges of Inquiry-Based Learning Through Technology and Curriculum Design. The Journal of the Learning Sciences, 8(3,4), pp. 391-450.

http://dx.doi.org/10.1080/10508406.1999.9672075

http://dx.doi.org/10.1207/s15327809jls0803\&4_3

Friel, S. N., Curcio, F. R. y Bright, G.W. (2001). Making sense of graphs: Critical factors influencing comprehension and instructional implications. Journal for Research in Mathematics Education, pp. 124-158.

http://dx.doi.org/10.2307/749671

Gil Pérez, D. (1994). Diez años de investigación en didáctica de las ciencias: realizaciones y perspectivas. Enseñanza de las Ciencias, 12(2), pp. 154-164.

Gil Pérez, D. y Valdés Castro, P. (1996). La orientación de las prácticas de laboratorio como investigación: un ejemplo ilustrativo. Enseñanza de las Ciencias, 14(2), pp. 155-163.

Hammer, D. (1997). Discovery learning and discovery teaching. Cognition and Instruction, pp. 15(4), 485-529. http://dx.doi.org/10.1207/s1532690xci1504_2

Hernández, C. A. (2001). Aproximación a un estado del arte de la enseñanza de las ciencias en Colombia. Estados del arte de la investigación en educación y pedagogía en Colombia. Bogotá: Icfes, Colciencias, Sicolpe.

Hernández, J. T., Figueroa, M., Carulla, C., Patiño, M. I., Tafur, M. y Duque, M. (2004). Pequeños Científicos, una aproximación sistémica al aprendizaje de las ciencias en la escuela. Revista de Estudios Sociales (019), pp. 51-56. 
Jaramillo, L. G. y García, J.C.A. (2004). La controversia Kuhn-Popper en torno al progreso científico y sus posibles aportes a la enseñanza de las ciencias. Cinta de Moebio, (20), pp. 1-13.

Kozma, R.B. (2000). The use of multiple representations and the social construction of understanding in chemistry. Innovations in science and mathematics education: Advanced designs for technologies of learning, pp. 11-46.

Kozma, R.B. (2003). The material features of multiple representations and their cognitive and social affordances for science understanding. Learning and Instruction, 13(2), pp. 205-226.

http://dx.doi.org/10.1016/S0959-4752(02)00021-X

Latour, B. (1987). Science in action: How to follow scientists and engineers through society. Cambridge, MA: Harvard University Press.

Latour, B. (1990). Drawing things together. The Map Reader, pp. 65-72.

Lederman, N. G. (1992). Students' and teachers' conceptions of the nature of science: A review of the research. Journal of Research in Science Teaching, 29(4), pp. 331-359. http://dx.doi.org/10.1002/tea.3660290404

Lehrer, R., \& Schauble, L. (2006). Scientific thinking and science literacy. En W. Damon, R. M. Lerner y Eliot-Pearson (eds.). Handbook of child psychology. Wiley Online Library.

LyNCH, M. (1988). The externalized retina: Selection and mathematization in the visual documentation of objects in the life sciences. Human Studies, 11(2), pp. 201-234.

Mankiewicz, R. (2005). Historia de las matemáticas: del cálculo al caos. Vol. 51. Paidos Iberica Ediciones SA.

MeIra, L. (1995). The microevolution of mathematical representations in children's activity. Cognition and Instruction, 13(2), pp. 269-313. http://dx.doi.org/10.1207/s1532690xci1302_5

Mellado Jiménez, V. (2003). Cambio didáctico del profesorado de ciencias experimentales y filosofía de la ciencia. Enseñanza de las Ciencias: revista de investigación y experiencias didácticas, 21(3), pp. 343-358.

Moya, A. y Campanario, J.M. (1999). ¿ Cómo enseñar ciencias? Principales tendencias y propuestas. Enseñanza de las Ciencias: revista de investigación y experiencias didácticas, 17(2), pp. 179-192.

National Research Council. Center for Science, M. a. E. E. (2000). Inquiry and the National Science Education Standards: A guide for teaching and learning. National Academies Press.

Oliva-Martínez, J.M. y Acevedo-Díaz, J.A. (2005). La enseñanza de las ciencias en primaria y secundaria hoy. Algunas propuestas de futuro. Revista Eureka sobre Enseñanza y Divulgación de las Ciencias(002), pp. 241-250.

Ortega, F.J.R. (2007). Modelos didácticos para la enseñanza de las ciencias naturales. Revista Latinoamericana de Estudios Educativos (Colombia) (2), pp. 41-60.

Perales Palacios, F.J. (2010). La reSolución de problemas en la didáctica de las ciencias experimentales. Revista Educación y Pedagogía, 10(21), pp. 119-143.

Roth, W.M. y McGinn, M.K. (1997). Graphing: Cognitive ability or practice? Science Education, 81(1), pp. 91-106.

http://dx.doi.org/10.1002/(SICI)1098-237X(199701)81:1<91::AID-SCE5>3.0.CO;2-X

Roth, W.M. y McGinn, M.K. (1998). Inscriptions: Toward a theory of representing as social practice. Review of educational research, 68(1), pp. 35-59. http://dx.doi.org/10.3102/00346543068001035

SANDOVAL, W.A. (2005). Understanding students' practical epistemologies and their influence on learning through inquiry. Science Education, 89(4), pp. 634-656.

http://dx.doi.org/10.1002/sce.20065 
SAndoval, W.A. y ReIser, B.J. (2004). Explanation-driven inquiry: Integrating conceptual and epistemic scaffolds for scientific inquiry. Science Education, 88(3), pp. 345-372.

http://dx.doi.org/10.1002/sce.10130

Spronken-Smith, R., Walker, R., Dickinson, K. J. M., Closs, G., Lord, J. y Harland, T. (2011). Redesigning a curriculum for inquiry: An ecology case study. Instructional Science, 39(5), pp. 721735.

http://dx.doi.org/10.1007/s11251-010-9150-5

Thomas, D. y Brown, J.S. (2011). A new culture of learning: cultivating the imagination for a world of constant change. Lexington, Ky: CreateSpace.

Trujillo de Figarella, E. (2001). Desarrollo de la actitud científica en niños de edad preescolar. Anales de la Universidad Metropolitana, 1(2), pp. 187-195.

Yoo, S. Y. (2010). Early Childhood Teachers' Empowerment and Implementation of Teaching Method Programs for Child Development in Science Education. Education, 130(4), pp. 556-560. 


\title{
If Galileo Galilei had had a digital camera: teaching science to a digital generation
}

\author{
Luis Alejandro Andrade Lotero \\ Indiana University, USA \\ laandrad@indiana.edu
}

To transcend a traditional type of education and in order to promote constructivist ways of teaching, in today's Sciences education it is commonly accepted that students ought to play a more central role (c.f., Gil Pérez, 1994; Mellado Jiménez, 2003; Moya \& Campanario, 1999). Among several alternative models, in the last decades learning through inquiry has gained momentum in the Spanish (c.f., Gil Pérez \& Valdés Castro, 1996; Moya \& Campanario, 1999; Ortega, 2007) as well as in the English speaking world (c.f., Abd-El-Khalick et al., 2004; Edelson, Gordin, \& Pea, 1999; National Research Council. Center for Science, 2000; Spronken-Smith et al., 2011). In particular, the inquiry model highlights laboratory practices as well as problem solving skills and empirical experimentation (Carrascosa, Pérez, Vilches, \& Valdez, 2008; Perales Palacios, 2010).

It is well agreed that measurement and representational tools are at the core of scientific practices (Kozma, 2000), tools that increasingly rely on new information technologies (Kozma, 2003). This technological trend has had an impact on educational practices, pushing educators' methods and goals to keep up to date with the digital world (Battro \& Denham, 1997; Thomas \& Brown, 2011). For instance, digital cameras -with which one can capture photos and video- appear to be a powerful, didactic tool in renovating middle and elementary school practices in Science education (Carrascosa et al., 2008).

The purpose of this paper is to show three feasible ways in which digital cameras can play a transformative role in the Science classroom. Steaming from our own experience with Childhood Education pre-service teachers, these three examples illustrate project-based, student-centered educational practices. However, we do not intend to exhaust the ample spectrum of possibilities this tool opens up, nor do we want to provide a fine-grain detailed sequence of the didactic practices. Instead, our purpose is to advocate that an inquiry-based education, underpinned in low-cost technologies, is indeed possible. We believe that the cost-benefit of such simple, yet powerful practices will reflect in highly motivated students and a new approach to support $21^{\text {st }}$ century skills. We believe this work might be useful to school teachers, from elementary to middle school years, as well as educational researchers interested in Science education, curriculum and instruction in the context of digital technologies in the classroom.

In this paper we provide a brief literature review of science education and how its history is intertwined with the practice of inquiry and emerging demands for a new culture of learning. We introduce three case studies for which the digital camera was the fulcrum around classroom practices were developed. The first case recreates the classic experiment by Galilei on the free fall of bodies, but with the digital camera it highlights a powerful way to capture empirical evidence from video and support later analysis with video-editing software. The second case recreates ancient astronomical observations in which a long sequence of photographs reveals the Sun as a celestial body worthy of inquiry. The third case empowers elementary students, who capture everyday objects through pictures that then become the center of their instruction. In the last section, we acknowledge further ways in which turning in the digital camera to the student can expand instructional practices as well as educational research. 